\title{
Atraumatic Restorative Treatment: A Short Review
}

\author{
M Vijaya Raju ${ }^{1}$, Vinay Darshan ${ }^{2}$, A.P Nirmal Raj ${ }^{3}$, Sanajay Kumar ${ }^{4}$, S. Revathi ${ }^{5}$ \\ ${ }^{1}$ Reader, Dept of Pedodontics \& Preventive Dentistry, College of Dental Sciences \& Hospital, Amargadh, Bhavnagar Dist, Gujarat, INDIA \\ ${ }^{2}$ Reader, Dept of Orthodontics, College of Dental Sciences \& Hospital, Amargadh, Bhavnagar Dist, Gujarat, INDIA \\ ${ }^{3} \mathrm{HOD}$, Dept of Prosthodontics, College of Dental Sciences \& Hospital, Amargadh, Bhavnagar Dist, Gujarat, INDIA \\ ${ }^{4}$ Senior Leccturer, Dept of Prosthodontics, College of Dental Sciences \& Hospital, Amargadh, Bhavnagar Dist, Gujarat, INDIA \\ ${ }^{5}$ Post Graduate Student, Dept Oral Pathology and Microbiology, Mahatama Gandhi Post Graduate Institute of Dental Sciences, Pondicherry, INDIA \\ Correspondence (Email): \\ faridi17@rediffmail.com
}

\begin{abstract}
In certain patients for whom there is increased demand for the treatment needs such as rural areas, an attempt has been made to improve the status of the oral health by use of the procedures which arrest the destructive dental caries process and alter microorganisms to favorable condition. This is called as atraumatic restorative treatment. It consists of low-cost and simplified technique using only hand instruments for the removal of the carious lesion and to arrest the progression of the caries. Since its discovery it has becoming an important treatment modality for the oral health care workers in managing dental caries. The present article reviews the importance of the atraumatic restorative treatment.
\end{abstract}

Key words: Atraumatic Restorative Treatment, Dental Caries, Hand Instruments

\begin{tabular}{ll}
\hline $6 / 30 / 2016$ & Source of Support: Nil, No Conflict of Interest: Declared \\
\hline $\begin{array}{l}\text { This article is is licensed under a Creative Commons Attribution-NonCommercial 4.0 International License. } \\
\text { Attribution-NonCommercial (CC BY-NC) license lets others remix, tweak, and build upon work non-commercially, and although the new works must also } \\
\text { acknowledge \& be non-commercial. }\end{array}$
\end{tabular}

\section{INTRODUCTION}

Dental caries is a microbial disease of the teeth damaging the tooth structure and resulting in the cavity formation in the hard tissues of the teeth (Dorri etal, 2009). The Atraumatic Restorative Treatment (ART) alternative was developed in the mid-1980s to provide dental restorative and preventive care to the patients in areas that are out of reach like rural areas (Stadart etal, 2012).

This method consists of the removing infected (soft demineralized carious) tooth tissue with only manual instruments, followed by the restoration of the cleaned cavity and associated pits and fissures with an adhesive restorative material. The resulting sealant-restoration is thus both preventive and restorative (Dorri etal, 2009), (Stadart etal, 2012). The restorative material is usually conventional glass polyalkenoate (ionomer) restorative cement (GIC). GICs demonstrate sustained fluoride release, pulpal biocompatibility, and chemical adhesion to tooth substance (Smales and Yip, 2000).

GIC creates a chemical bond between the cement and the remaining enamel and dentine. This adherence provides an adaptive seal, and, as the material slowly leaches fluoride ions into the adjacent tooth tissue, GICs are capable of halting or slowing the progression of carious lesions (Sajjanshetty etal, 2013). With the launch of high-viscosity glassionomers for use with ART sealants, the retention rate of glass-ionomer sealants has increased substantially in comparison to that of the low- and medium-viscosity glass-ionomers previously used (Frencken and Holmgren, 2014).

Advantages (Dorri etal, 2009), (Stadart etal, 2012), (Smales and Yip, 2000), (Holmgren etal, 2013), (Lopez etal, 2005):

- The minimally-invasive procedure.

- Pain-free and readily accepted by children.

- Management of early childhood caries.

- Reduced risk for subsequent endodontics and tooth extraction.

- Lower dental anxiety in children and adults (more patient-friendly).

- This approach requires neither electricity nor running water and can therefore be applied in almost any setting. 
- The use of available \& inexpensive hand instruments rather than more expensive electrically-driven dental equipment.

- Conservation of sound tooth tissue through the chemical adhesion of glass ionomer.

- Suitable for patients with permanent or temporary physical disabilities.

Indications (Holmgren etal, 2013), (Lopez etal, 2005), (Bhat and Kumar, 2011):

- Very young children who are being introduced to oral care.

- Patients who experience extreme fear or anxiety about dental procedures.

- Cavity which is accessible to hand instruments.

- Mentally and/or physically handicapped patients.

- Home-bound elderly and residents of nursing homes.

- Cavity involving the dentine.

Contraindications (Holmgren etal, 2013), (Bhat and Kumar, 2011):

- Presence of swelling (abscess) or fistula (opening from abscess to the oral cavity) near the carious tooth.

- If the pulp of the tooth is exposed.

- Painful teeth for a long time.

- Opening of the carious cavity inaccessible to hand instruments.

- Clear signs of a cavity, for example in a proximal surface, but the cavity cannot be entered from the proximal or the occlusal directions.

Using ART, dentists can provide restorative care to patients in the dental clinic even if conventional equipment is unavailable or out-of-order, and in outreach situations. ART does not rely on electricity and piped water systems which are required for conventional rotary restorative treatment procedures (Shenoy etal, 2014). Among the reasons given for avoiding dental treatment, the most important were those related to the patient's comfort, use of local anesthetic and the noisy drill and suction. The most favorable aspects of the ART approach is to change the patients' expressions from fearful to more relaxed (Shenoy etal, 2014). Due to the "atraumatic" aspects, the ART approach may be a very useful treatment for the pregnant women (Shenoy etal, 2014).

Because of its chemical adhesion to enamel and its improved physical properties, high-viscosity glass ionomer cement can also be used to seal pits and fissures in the absence of cavitation. These are called ART sealants. After cleaning the surface, high-viscosity glass ionomer cement is pressed into the pits and fissures with an index finger. Thus the ART approach consists of two components; a sealant restoration and a preventive sealant (Stadart etal, 2012).

Recently, modified ART approaches have been introduced, as opposed to 'true' ART, which was described above. These modified approaches involve opening the cavity with a drill, cleaning, restoring and finishing with hand instruments, or using alternative restorative materials including amalgam (Dorri etal, 2009).

\section{CONCLUSION}

Atraumatic restorative treatment is very helpful for the rural patients, pregnant woman's or other fearful patients. Thus every dental practitioner should be aware of Atraumatic restorative treatment. So that maximum patients can take benefit from this treatment approach.

\section{REFERENCES}

Bhat PK, Kumar A. Atraumatic Restorative Treatment - A rural prospective. Journal of Health Sciences and Research 2011; 2(1):29-32.

Dorri M, Sheiham A, Marinho VCC. Atraumatic restorative treatment versus conventional restorative treatment for the management of dental caries. Cochrane Database of Systematic Reviews 2009; 4:1-10.

Frencken JE, Holmgren CJJ. Caries management through the Atraumatic Restorative Treatment (ART) approach and glassionomers: update 2013. Braz Oral Res (Sao Paulo) 2014; 28(1):1-4.

Holmgren CJ, Roux D, Domejean S. Minimal intervention dentistry: part 5. Atraumatic restorative treatment (ART) - a minimum intervention and minimally invasive approach for the management of dental caries. British Dental Journal 2013; 214(1):11-8.

Lopez N, Simpser-Rafalin S, Berthold P. Atraumatic Restorative Treatment for Prevention and Treatment of Caries in an Underserved Community. Am J Public Health 2005; 95:1338-9.

Sajjanshetty S, Hugar D, Jain D, Soujanya KP, Khan MI. Atraumatic restorative treatment -a review. Journal of Evolution of Medical and Dental Sciences 2013; 2(3):235-9.

Shenoy R, Jain A, Kundabala M. Atraumatic restorative treatment for dental caries among pregnant women attending primary health centres: A small scale demonstration. J Interdiscip Dentistry 2014; 4:71-5.

Smales RJ, Yip HK. The atraumatic restorative treatment (ART) approach for primary teeth: review of literature. American Academy of Pediatric Dentistry 2000; 22(4):294-8.

Stadart L, Franca GD, Colares V. Atraumatic Restorative Treatment in permanent molars: a systematic review. Braz J Oral Sci 2012; 11(1):38-41.

$$
--0 \text {-- }
$$

